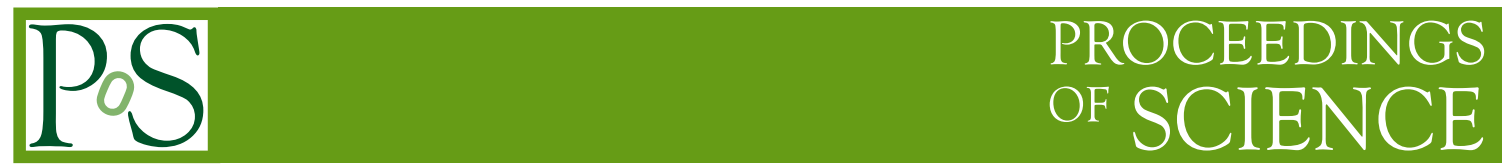

\title{
Determination of $\kappa_{\mathrm{t}}$ from $\mathrm{t} \overline{\mathrm{t}}$, $\mathrm{t} \overline{\mathrm{t}} \overline{\mathrm{t}}$, and others
}

\section{Emanuele Usai on behalf of the ATLAS and CMS Collaborations ${ }^{a, *}$}

${ }^{a}$ Department of Physics, Brown University,

182 Hope Street, Providence, RI, USA

E-mail: emanuele.usai@cern.ch

In this document I report the determination of the Yukawa coupling of the top quark in $\mathrm{t} \overline{\mathrm{t}}$ and $\mathrm{t} \overline{\mathrm{t}} \overline{\mathrm{t}}$ final states in ATLAS and CMS. I will also briefly describe the determination of $\kappa_{\mathrm{t}}$ and its CP structure from $\mathrm{t} \overline{\mathrm{t}} \mathrm{H}$ final states.

The Ninth Annual Conference on Large Hadron Collider Physics - LHCP2021

7-12 June 2021

Online

${ }^{*}$ Speaker 


\section{Introduction}

In the standard model (SM) the Yukawa couplings are proportional to the mass of the fermions. Being the heaviest particle in the SM, the top quark has the largest Yukawa coupling to the Higgs boson $\left(\kappa_{\mathrm{t}}\right)$, therefore it provides an insight on SM physics in the Higgs sector. It is also particularly sensitive to some extensions of the standard model. Such extensions would alter the top quark-Higgs interaction and therefore modify the value of $\kappa_{\mathrm{t}}$ in measurable ways. The $\kappa_{\mathrm{t}}$ parameter can be probed directly, that is in events containing both the top quark and the Higgs boson in the final state, e.g. in t $\mathrm{t} \mathrm{H}$ and tH events. $\kappa_{\mathrm{t}}$ can also be measured indirectly in final states with only the Higgs boson or top quarks. Examples of these measurements are analyses probing the $\bar{t} \bar{t}$ and $\mathrm{t} t \bar{t} t$ final states. In the case of $t \bar{t}$, the coupling to the Higgs boson is exposed through loop diagrams, while in the case of four-top-quark production, one of the contributing tree-level diagrams includes the Higgs boson. Therefore, deviations of $\kappa_{\mathrm{t}}$ from unity are indicative of new physics.

The different final states used to probe $\kappa_{\mathrm{t}}$ yield different kind of information. For example, the measurement of $\kappa_{\mathrm{t}}$ from $\mathrm{tt} H$ events is not sensitive to its sign. However, due to the interference between the $\mathrm{W}$ boson and top quark couplings, the tH final state is sensitive to the sign of $\kappa_{\mathrm{t}}$. More generally, the CP properties of the Higgs boson can be probed in both t $\mathrm{t} \mathrm{H}$ and tH modes.

\section{2. $\kappa_{\mathrm{t}}$ from $\mathrm{t} \overline{\mathrm{t}}$}

The CMS experiment [1] measured the top quark Yukawa coupling using kinematic distributions of $t \bar{t}$ events in the final state with two leptons [2]. This analysis includes data collected by the CMS experiment at $13 \mathrm{TeV}$ during the entire Run $2\left(137 \mathrm{fb}^{-1}\right)$.

As briefly mentioned in Sec.1, Feynman diagrams containing the Higgs boson contribute to $\mathrm{t} \overline{\mathrm{t}}$ production at order $\alpha_{s}$ times the Yukawa coupling. This yields small effects on the total cross section, but has a noticeable impact on the differential distributions of kinematic variables. This shape effect is enhanced in the case of an anomalously large coupling.

In this analysis, events with two leptons are selected: ee, e $\mu$ and $\mu \mu$. In single-flavor channels, $\mu \mu$ and ee, selection criteria on the invariant mass of the dilepton pair and on missing transverse momentum are added to reduce the background from Drell-Yan events.

In principle, the invariant mass of the $\bar{t} \bar{t}$ system and the difference in the top quark rapidity are particularly sensitive to variations of the Yukawa coupling. These differences can be exploited to derive multiplicative correction factors to reweight the full detector simulation to a specific value of the Yukawa coupling. The strategy of the analysis consists in performing a profile likelihood scan on the value of $\kappa_{\mathrm{t}}$, the Yukawa coupling normalized to the SM value, to extract the best fit value of the coupling.

In terms of event reconstruction, the dilepton t⿱亠幺 system can usually be reconstructed in good approximation, but is very sensitive to the missing transverse momentum and its uncertainties, particularly the off-shell component. Therefore the analysis uses proxy substitute variables that do not include the missing transverse momentum. These variables are used to derive and apply the correction factors to the simulation in place of the original $t \bar{t}$ system variables. The proxy variables maintain the sensitivity to variations of the Yukawa coupling. A profile likelihood scan for $\kappa_{\mathrm{t}}$ is 
performed showing the best fit value to be $\kappa_{\mathrm{t}}=1.16_{-0.08}^{+0.07}$ (stat $)_{-0.27}^{+0.17}$ (syst). Additionally, the coupling is constrained to be smaller than 1.62 at $95 \%$ confidence level (CL).

\section{3. $\kappa_{t}$ from $t \bar{t} t \bar{t}$}

The production of four top quarks (t $\mathrm{t} t \mathrm{t} t)$ is a rare process in the SM, the computed cross section at next-to-leading order (NLO) in quantum chromodynamics (QCD) plus electroweak (EWK) is $12 \mathrm{fb}$ [3]. This is approximately one order of magnitude smaller than the cross section of Higgs boson production in association with top quarks $(\mathrm{tt} H)$. About $20 \%$ of the production diagrams for $t \bar{t} t \bar{t}$ contain the Higgs boson, hence the top quark-Higgs boson coupling has a substantial influence on the value of the cross section of tttti. The process has not been observed yet, but the ATLAS experiment [4] has shown evidence of tttt production at the LHC [5].

The CMS Collaboration conducted a search for four-top-quark production in final states with two same-sign or multiple leptons with the full Run 2 data set [6]. The tittt process produces a challenging signature in the detector: the final state considered in the CMS analysis is characterized by two leptons, 4 b-flavor jets (b jets) and 4 light-flavor jets (light jets), or three leptons, $4 \mathrm{~b}$ jets and 2 light jets, or four leptons, $4 \mathrm{~b}$ jets and no light jets. The branching fraction of $t \overline{t t} \bar{t}$ for two, three and four leptons in the final state is $0.28,0.09$, and 0.04 , respectively.

In this final state the main background processes are $\overline{\mathrm{t}} \mathrm{H}, \overline{\mathrm{t}} \mathrm{Z}, \mathrm{t} \overline{\mathrm{t}} \mathrm{W}$, and events with a misidentified lepton. The analysis uses a boosted decision tree (BDT) to distinguish the four-top-quark signal from the dominant t⿳亠丷厂 background. This leads to a substantial improvement over the traditional cut and count approach. The analysis phase space is subdivided in fourteen signal regions and two separated control regions enriched in events with $\mathrm{W}$ and $\mathrm{Z}$ bosons. The events are categorized using a combination of selection criteria on the number of leptons, jet multiplicity, and $b$ jet multiplicity.

The analysis measures the four-top-quark cross section to be $\sigma_{\text {titti }}=12.6_{-5.2}^{+5.8} \mathrm{fb}$. The BDT analysis reaches 2.6 standard deviations significance (2.7 expected from simulation) over the no $t \bar{t} t \bar{t}$ hypothesis. A search for $t \bar{t} t \bar{t}$ from the ATLAS Collaboration using single lepton, dilepton and multi lepton final states and using the full Run 2 data set reached a 4.7 standard deviations significance (2.6 expected from simulated events) over the null hypothesis [5]. The best estimate for the cross section is $\sigma_{\text {tittĩ }}=24_{-6}^{+7} \mathrm{fb}$.

In the CMS analysis, the dependence of the four-top-quark cross section on $\kappa_{\mathrm{t}}$ is obtained from Cao et al. [7] at leading order and scaled to the measured cross section value of $12.6 \mathrm{fb}$. As mentioned previously, $\mathrm{t} \mathrm{t} \mathrm{H}$ is a relevant background for this analysis and it is affected by the variation of $\kappa_{\mathrm{t}}$. As a consequence of this, the measured cross section does have a mild dependence on the assumed value of $\kappa_{\mathrm{t}}$. The parametrization of the cross section as a function of $\kappa_{\mathrm{t}}$ allows to extract an upper limit on the value of the Yukawa coupling. In the most conservative scenario, that is taking into account he effect of $\kappa_{\mathrm{t}}$ variation in the t⿱亠幺十H background, the ratio of the Yukawa coupling to the SM value is found to be $\kappa_{\mathrm{t}}<1.7$ at $95 \% \mathrm{CL}$. When assuming on-shell Yukawa coupling to be the same as the SM, that is with no rescaling of the $\mathrm{tt}_{\mathrm{t}} \mathrm{H}$ process, the ratio of the off-shell Yukawa coupling to the SM value is found to be smaller than 1.8 at $95 \%$ CL. While this result is more stringent than other measurements, it does not make any assumption of the width of the Higgs boson. 


\section{4. $\kappa_{t}$ from $t \bar{t} H$}

The ATLAS Collaboration performed a measurement of the CP properties of Higgs boson interactions using $\mathrm{t} \overline{\mathrm{t}} \mathrm{H}$ and $\mathrm{tH}$ production modes and the decay of the Higgs boson to a pair of photons [8]. The analysis uses two separate BDTs, one to discriminate $\bar{t} \mathrm{H}$ and tH signal from backgrounds and another to distinguish CP-odd from CP-even Higgs-top quark couplings. Constraints on $\kappa_{\mathrm{t}}$ are extracted from a bidimensional fit to $\kappa_{\mathrm{t}} \cos \alpha$ and $\kappa_{\mathrm{t}} \sin \alpha$, the CP-even and CP-odd components of $\kappa_{\mathrm{t}}$, respectively, where $\alpha$ is defined as the mixing angle between the two components. Limits on the value of the mixing angle $\alpha$ are extracted: $|\alpha|>43$ is excluded at $95 \% \mathrm{CL}$ with no assumptions on $\kappa_{\mathrm{t}}$. Additionally, $\alpha=90,180$ are excluded at 3.9 and $2.5 \sigma$. CMS performed a measurement in a similar final state and excludes a pure CP-odd structure of the top quark Yukawa coupling at 3.2 $\sigma$ [9], that is to be compared to the $\alpha=90$ case from the correspondent ATLAS analysis.

CMS measured $\kappa_{\mathrm{t}}$ from $\mathrm{t} \overline{\mathrm{t}} \mathrm{H}$ events in final states with electrons, muons, and hadronically decaying $\tau$ leptons using the full Run2 data set [10]. This analysis uses different BDTs and Deep Neural Networks (DNNs) to enhance background rejection. In order to extract a limit on $\kappa_{\mathrm{t}}$, the production rates of $\mathrm{t} \overline{\mathrm{t}} \mathrm{H}$ and $\mathrm{tH}$ are parametrized in function of the Yukawa coupling. In particular, in the case of tH events, varying $\kappa_{\mathrm{t}}$ affects the interference of the production diagrams for this process. This effect alters the kinematics of the events and is taken into account in the yields and in the shape of the distribution of BDT and DNN discriminators. The effect of the modified kinematics is taken into account in the acceptance of the selection criteria as well. The compatibility of the data with the various kt hypotheses is shown in the distribution of the log likelihood in function of kt and $\mathrm{kv}$, the coupling to vector bosons, which can affect kinematics as well. The analysis measures $-0.9<\kappa_{\mathrm{t}}<-0.7$ and $0.7<\kappa_{\mathrm{t}}<1.1$ at $95 \%$ confidence level, compatible with SM.

Finally, both ATLAS and CMS provide an estimate of $\kappa_{\mathrm{t}}$ from combined measurements of the Higgs boson in multiple final states and production modes. In this context, ATLAS measures $\kappa_{\mathrm{t}}=0.96 \pm 0.08$ [11]. In this combined measurement using it can be noted that the ZH production mode contributes to the determination of $\kappa_{\mathrm{t}}$ as well. The CMS experiment, using a partial Run2 data set combination measures $\kappa_{\mathrm{t}}=1.01 \pm 0.11$ [12].

\section{Conclusions}

It has been shown how $\kappa_{\mathrm{t}}$ and the $\mathrm{CP}$ structure are measured across different final states with and without the Higgs boson in the final state. Each measurement provides a different and complementary information. The $\kappa_{\mathrm{t}}$ parameter is extracted from $\mathrm{t} \overline{\mathrm{t}}$ events by CMS using the full Run 2 data set. The value is $\kappa_{\mathrm{t}}=1.16_{-0.08}^{+0.07}(\mathrm{stat})_{-0.27}^{+0.17}$ (syst). The Yukawa coupling of the top quark is measured to be $\left|\kappa_{\mathrm{t}}\right|<1.7$ in titt events using the full Run2 data set from the CMS Experiment. The measurement is independent of the total width of the Higgs boson. The CP structure of the top quark-Higgs boson coupling $\mathrm{CP}$ is measured in $\mathrm{tt} H$ and $\mathrm{tH}$ events with $\mathrm{H} \rightarrow \gamma \gamma$ by ATLAS and CMS and a pure CP-odd coupling is excluded at $3.9 \sigma$ and $3.2 \sigma$, respectively. Finally, $\kappa_{\mathrm{t}}$ is measured in $\mathrm{t} \mathrm{t} H$ and tH events with $\mathrm{H} \rightarrow \mathrm{WW}, \mathrm{ZZ}, \tau_{h} \tau_{h}$ by CMS $-0.9<\kappa_{\mathrm{t}}<-0.7$ and $0.7<\kappa_{\mathrm{t}}<1.1$ at $95 \%$ confidence level. 


\section{References}

[1] The CMS Collaboration. The CMS experiment at the CERN LHC. Journal of Instrumentation, 3(08):S08004-S08004, 2008.

[2] The CMS Collaboration. Measurement of the top quark Yukawa coupling from tt kinematic distributions in the dilepton final state in proton-proton collisions at $\sqrt{s}=13 \mathrm{TeV}$. Phys. Rev. D, 102(9):092013, 2020.

[3] Rikkert Frederix, Davide Pagani, and Marco Zaro. Large NLO corrections in $t \bar{t} W^{ \pm}$and $t \bar{t} t \bar{t}$ hadroproduction from supposedly subleading EW contributions. JHEP, 02:031, 2018.

[4] The ATLAS Collaboration. The ATLAS experiment at the CERN large hadron collider. Journal of Instrumentation, 3(08):S08003-S08003, 2008.

[5] The ATLAS Collaboration. Measurement of the $t \bar{t} t \bar{t}$ production cross section in $p p$ collisions at $\sqrt{s}=13 \mathrm{TeV}$ with the ATLAS detector. arXiv:2106.11683, 2021.

[6] The CMS Collaboration. Search for production of four top quarks in final states with same-sign or multiple leptons in proton-proton collisions at $\sqrt{s}=13 \mathrm{TeV}$. Eur. Phys. J. C, 80(2):75, 2020.

[7] Qing-Hong Cao, Shao-Long Chen, and Yandong Liu. Probing Higgs Width and Top Quark Yukawa Coupling from $t \bar{t} H$ and $t \bar{t} t \bar{t}$ Productions. Phys. Rev. D, 95(5):053004, 2017.

[8] The ATLAS Collaboration. CP Properties of Higgs Boson Interactions with Top Quarks in the $t \bar{t} H$ and $t H$ Processes Using $H \rightarrow \gamma \gamma$ with the ATLAS Detector. Phys. Rev. Lett., 125(6):061802, 2020.

[9] The CMS Collaboration. Measurements of $\mathrm{t} t \bar{H}$ Production and the CP Structure of the Yukawa Interaction between the Higgs Boson and Top Quark in the Diphoton Decay Channel. Phys. Rev. Lett., 125(6):061801, 2020.

[10] The CMS Collaboration. Measurement of the Higgs boson production rate in association with top quarks in final states with electrons, muons, and hadronically decaying tau leptons at $\sqrt{s}=$ 13 TeV. Eur. Phys. J. C, 81(4):378, 2021.

[11] The ATLAS Collaboration. A combination of measurements of Higgs boson production and decay using up to $139 \mathrm{fb}^{-1}$ of proton-proton collision data at $\sqrt{s}=13 \mathrm{TeV}$ collected with the ATLAS experiment. Technical report, CERN, Geneva, Aug 2020. ATLAS-CONF-2020-027, https://cds.cern.ch/record/2725733.

[12] The CMS Collaboration. Combined Higgs boson production and decay measurements with up to $137 \mathrm{fb}^{-1}$ of proton-proton collision data at $\sqrt{s}=13 \mathrm{TeV}$. Technical report, CERN, Geneva, 2020. CMS-PAS-HIG-19-005, http://cds.cern.ch/record/2706103. 